\title{
A novel PDCDI0 gene mutation in cerebral cavernous malformations: a case report and review of the literature
}

This article was published in the following Dove Press journal:

Journal of Pain Research

\section{Weiwei Yu \\ Haiqiang Jin \\ Qian You \\ Ding Nan \\ Yining Huang}

Department of Neurology, Peking University First Hospital, Beijing 100034 ,

People's Republic of China
Correspondence: Yining Huang Department of Neurology, Peking University First Hospital, 8 Xishiku Street, Xicheng District, Beijing 100034, People's Republic of China

Email ynhuang@bjmu.edu.cn

\begin{abstract}
Cerebral cavernous malformations (CCMs) are one of the most common types of vascular malformation, which are featured enlarged and irregular small blood vessels. The cavernous cavities are merely composed of a single layer of endothelial cells and lack other support tissues, such as elastic fibers and smooth muscle, which make them elastic. CCMs may develop in sporadic or familial forms with autosomal dominant inheritance. Mutations have been identified in three genes: KRIT1, MGC4607, and PDCD10. Here, we report a typical case of CCMs in a 44-year-old woman associated with a novel mutation in PDCD10 gene. The patient, diagnosed with CCMs, has been suffering from headache for several months. Analyses of the Whole Exome Sequencing revealed a novel diseaseassociated mutation in the already known disease-associated PDCD10 gene. This mutation consists a nucleotide deletion (c.212delG) within the exon 4, resulting in premature protein termination (p.S71Tfs*18). This novel mutation significantly enriches the spectrum of mutations responsible for CCMs, providing a new evidence for further clarifying the genotype-phenotype correlations in CCMs patients.
\end{abstract}

Keywords: cerebral cavernous malformations, hemorrhage, subcutaneous nodules, PDCD10 gene, frameshift mutation

\section{Introduction}

Cerebral cavernous malformations (CCMs) are one of the most common types of vascular malformation. The prevalence rate in the general population has been estimated $0.4-0.6 \%^{1}$ while it represents $5-15 \%$ of all vascular malformations of the central nervous system $(\mathrm{CNS}){ }^{2} \mathrm{CCMs}$ are featured enlarged and irregular small blood vessel. The cavernous cavities merely composed of a single layer of endothelial cells lack some support tissues, such as elastic fibers and smooth muscle, thus making vessel walls fragile and immature. ${ }^{3,4}$ Because of the abnormally thin walls, the blood vessels are prone to recurrent hemorrhages that can cause various nervous system diseases, such as chronic headaches, epileptic seizures and stroke-like symptoms. ${ }^{5}$ However, only $20-30 \%$ of individuals with CCMs experience some related health problems. Generally, CCMs predominantly affect the CNS, but that sometimes affect other vital tissues, including the retina, skin, ${ }^{6}$ and even liver. ${ }^{7}$

Magnetic resonance imaging (MRI) has been historically regarded as the gold standard for CCMs diagnosis according to clinical guidelines. ${ }^{8}$ Compared with computed tomography (CT), MRI possesses much more sensitivity and specificity 
toward the diagnosis of CCMs lesions, especially when gradient echo T2*-weighted (GRE T2*) and susceptibilityweighted imaging (SWI) sequences are utilized, ${ }^{9}$ achieving an increased reported incidence of $\mathrm{CCMs}$ in the general population. ${ }^{10}$ T2-weighted images reveal that lesions in patients with CCMs who had experienced repeated bleedings characterized by mulberry- or popcornlike appearance and a dark rim due to hemosiderin deposition.

CCMs can develop in both sporadic and familial forms. The pattern of inheritance of the familial form is autosomal dominant. Usually the familial cases typically exhibit multiple lesions, whereas sporadic cases usually have a single lesion. ${ }^{11}$ However, patients with no positive family history can also suffer from multiple lesions, ${ }^{12}$ which may result from a de novo mutation or a mutation inherited from an asymptomatic parent. ${ }^{13,14}$ Until now, mutations in three CCM genes, CCM1/krevl interaction trapped gene 1 (KRIT1), ${ }^{15,16}$ CCM2/MGC4607, ${ }^{17}$ and CCM3/programmed cell death $10(P D C D 10),{ }^{18,19}$ have been identified. More than $90 \%$ of patients with autosomal dominant CCMs and over $60 \%$ for isolated cases with multiple malformations carry the loss of function mutations in one of the three genes. ${ }^{20}$ About 200 distinct CCM1-CCM3 mutations have been identified in familial CCMs to date. ${ }^{21} P D C D 10 / C C M 3$ has recently been found and its mutations cause $10-15 \%$ of familial CCMs. ${ }^{22}$ According to the genotype-phenotype correlation analysis, patients with $P D C D 10 / C C M 3$ mutation may have a significant trend toward an earlier age at symptoms' onset ( $<15$ years old), and a higher risk of cerebral hemorrhage during childhood. ${ }^{23,24}$

Here, we report a novel PDCD10 frameshift mutation, detected in a heterozygous condition in a 44-year-old woman with CCMs. The study was conducted in accordance with the Ethics Committee of Peking University First Hospital and the case details were approved by the Ethics Committee of Peking University First Hospital to be published. Written informed consent was obtained from the patient to publish the case details and any accompanying images in medical journals.

\section{Case presentation}

A 44-year-old previously healthy woman presented to outpatient clinic with a 4-month history of progressively worsening headaches and dizziness, sometimes accompanied by vertigo, nausea, and vomiting. Headache was transiently controlled with trazodone, nigral, and paroxetine. Nonetheless, the headache was aggravated and ranged from the top of the head to the bilateral ankle and posterior occipital now, which seriously affected her sleeping. The patient did not have the history of hypertension, diabetes and psychosis, as well as the similar family history. Besides, the patient has a 7-yearhistory of multiple asymptomatic subcutaneous nodules located on right posterior occipital region (Figure 1A-B; red arrow) and the left posterior shoulder. Two years ago, the similar lesions subsequently appeared in the right nasal wing (Figure 1C; red arrow) and the right forehead. Skin examination found that the diameter of multiple nodular skin lesions was about $1 \mathrm{~cm}$ and their shape in T1WI sequence was oval with uniform low signal (Figure 1A; red arrow) while nearly round with popcorn-like appearance inside in T2WI sequence (Figure 1B; red arrow). The color of the overlying skin showed a very slightly bluish hue (Figure 1C) without any redness and swelling. The nodular mass was hard and no tenderness. By neurologic examination, it was shown that comprehension and computational abilities were slightly reduced. Near vision of the left eye is $\mathrm{Jr} 5$ and right eye $\mathrm{Jr} 4$. The right knee reflex was more active than the contralateral side. Both the bilateral palmar reflex and left side Rossolimo sign were positive. The head-MRI, including T2 Weight-sequence (Figure 2A), diffusion-weighted imaging-sequence (Figure 2B) and SWIsequence in 2017 (Figure 2C) showed multiple abnormally low signals in the brain characterized by mulberry-appearance with a dark rim or oval shape, which were considered as micro-hemorrhagic lesions. The head-MRI SWI sequence from the re-examination at the local hospital in 2018 showed increased microhemorrhagic lesion number (Figure 2D, blue arrows) and extension of the lesion size (Figure 2D, red arrows) at the same position in the brain, involving the cerebral hemispheres, cerebellum, and brain stem. That is the reason why the patient had progressively worsening headaches. Additionally, SWI in 2018 showed the obvious filled veins than before (Figure 2D, red boxes). Head CT showed some high-density lesions in the left semi-oval center and the left basal ganglia, which were considered as bleeding. Unfortunately, none of these family members could be contacted and investigated for the presence of CCMs.

We extracted DNA from peripheral blood of the patient using DNA Isolation Kit (Bioteke, AU1802). DNA libraries were prepared with KAPA Library Preparation Kit (Kapa Biosystems, KR0453) following the manufacturer's instructions. And sample dilution, flowcell loading, and sequencing were performed according to the Illumina specifications. Whole exons were sequenced on the HiSeq2500 platform as paired-end 200-bp reads. The sequencing data was evaluated by Illumina Sequence 

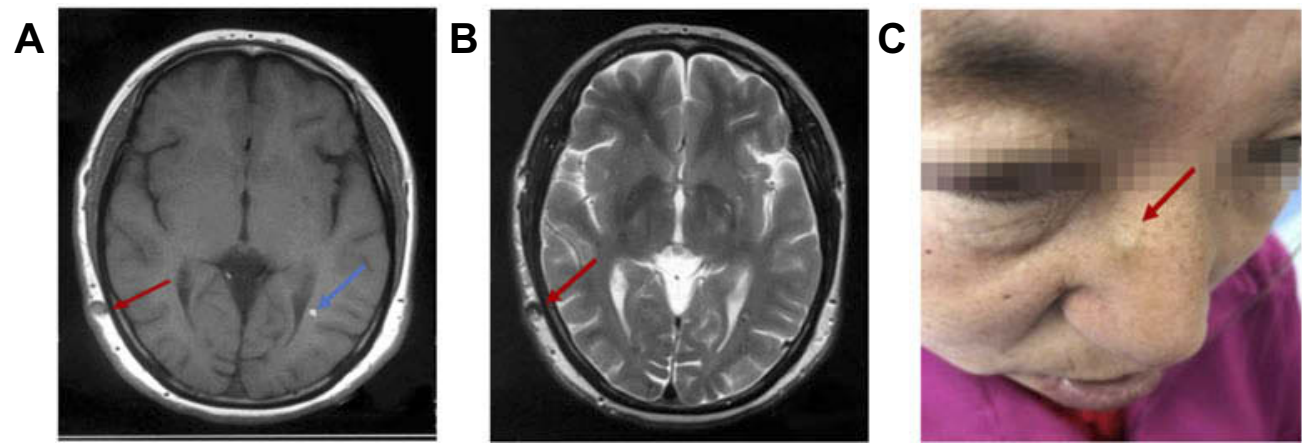

Figure I Representative subcutaneous nodular lesions of the patient. (A) The TI Weight-sequence showed the subcutaneous nodules on right posterior occipital region with oval and uniform low signal (red arrow) and the new lesion in the brain (blue arrow). (B) The T2 Weight-sequence revealed the nodular skin lesions on right occipital with nearly round low signal and popcorn-like appearance inside (red arrow); (C) the nodular skin lesions on right nasal wing (red arrow).

Control Software to remove adapter sequences in the raw data and discard low-quality reads with low base quality. Finally, sequencing analysis of the patient's whole exomic DNA revealed a novel disease-associated mutation in exon 4 of the already known disease-associated PDCD10 gene. It is a frameshift mutation in the heterozygous state: the
G nucleotide deletion at codon 212 position (Figure 3). According to HGMDpro database, the mutation site c. $212 \mathrm{delG}$ has not been reported so far. Therefore, it is a novel mutation. The mutation leads to premature protein termination (p.S71Tfs*18). Effects of the novel mutation on PDCD10 tertiary structure were predicted by RaptorX
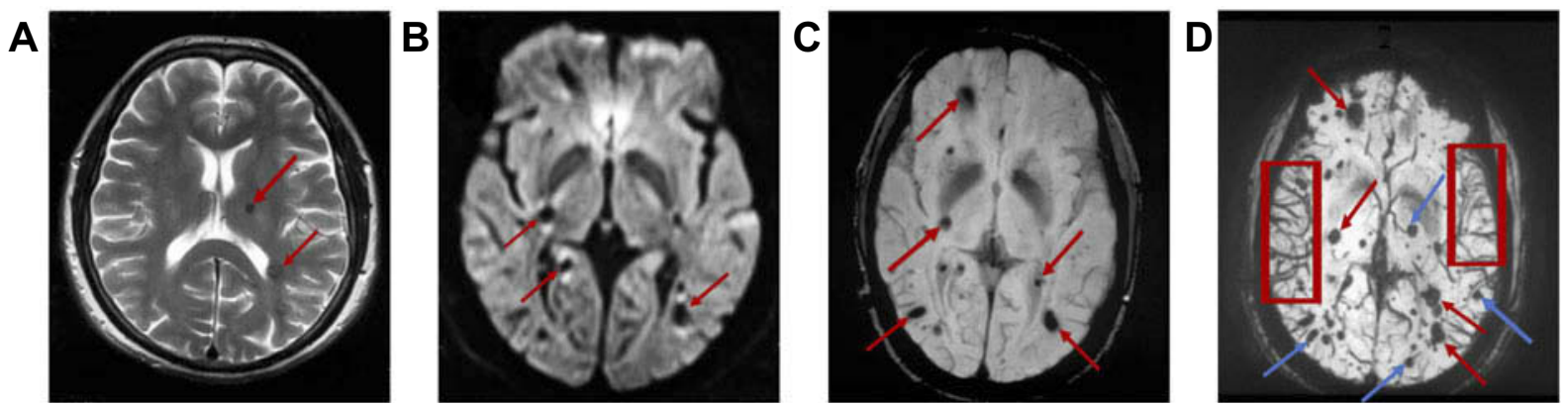

Figure 2 Representative cranial MRI of the patient. (A) The T2WI sequence showed the lesions were in round shape (long red arrow) or characterized by mulberryappearance and a dark rim (short red arrow); (B) some low signal lesions in the diffusion-weighted imaging (DWI)-sequence were characterized by high signals around (red arrows); (C) the susceptibility-weighted imaging (SWI)-sequence (TR:19,TE:25) in 2017 revealed multiple oval or round lesions; (D) the SWI (TR:20,TE:26) in 20I8 showed the old lesions in 2017 (Figure 2C; red arrows) had bigger diameters (red arrows) and the new low signal lesions (blue arrows) in the whole brain (cerebral hemispheres, cerebellum, and brain stem). The red box represented the filled veins.

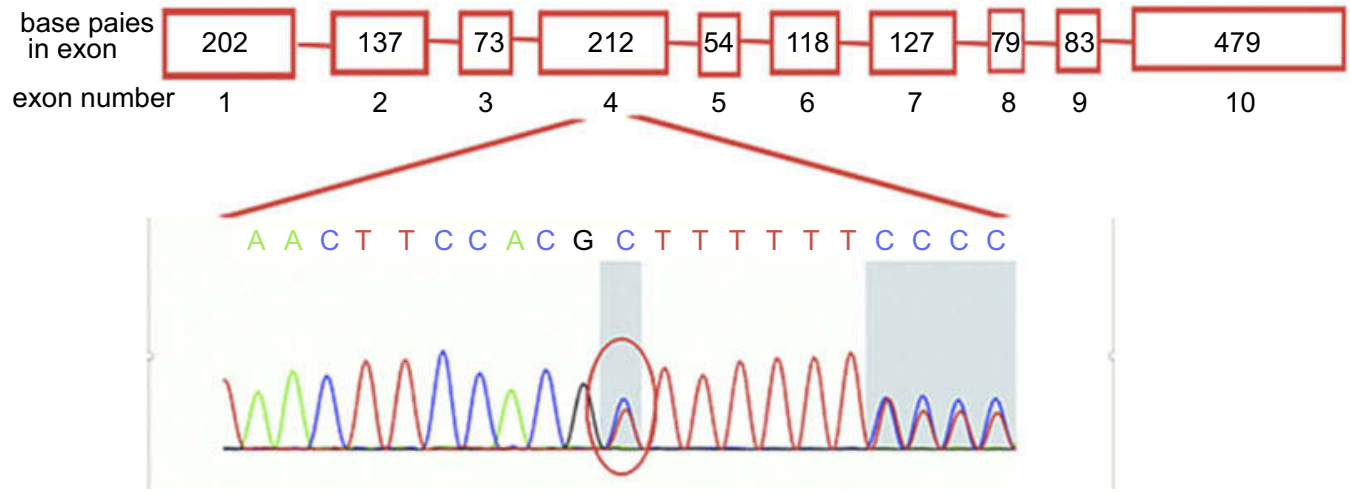

Figure 3 Graph showing PDCDIO coding sequence and exon4 sequence analysis. Exons are represented graphically as boxes with the exon number below the boxes, the size of each exon is shown as the number of base pairs directly inside each box. Sequencing with forward primer shows a heterozygous frameshift mutation: the $\mathrm{G}$ nucleotide deletion at codon $2 \mathrm{I} 2$ position (red circle). The frameshift mutation (c.2I2delG) led to premature protein termination. 

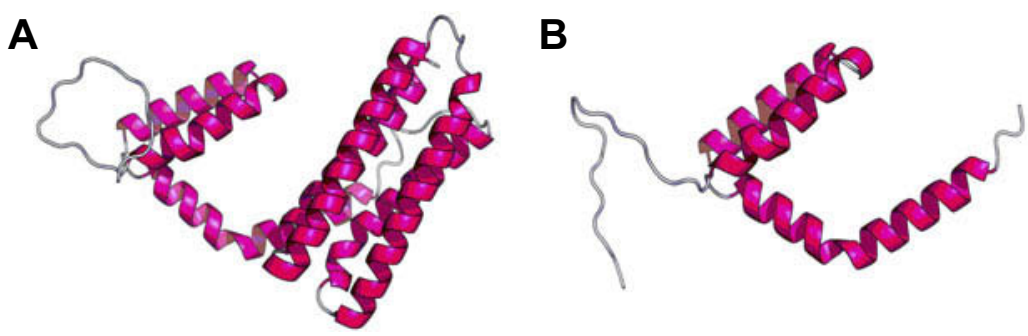

Figure 4 PDCDI0 Tertiary structure alteration prediction by RaptorX tool. (A) Tertiary structure of wild-type protein. (B) Tertiary structure of p.S7ITfs* 8 affected protein.

prediction tool. ${ }^{25}$ The results reveal tertiary structure change in mutated protein (Figure 4B) compared to wildtype one (Figure $4 \mathrm{~A}$ ). And there are no mutations were identified when analysis of KRIT1 and MGC4607 genes was performed.

\section{Discussion}

CCMs are vascular lesions of the CNS characterized by abnormally enlarged capillary cavities. ${ }^{26} \mathrm{CCMs}$ can arise anywhere in the body, especially in the forebrain, ${ }^{27}$ which can lead to focal neurological deficits, seizures, and hemorrhagic stroke. However, there is no effective pharmacologic therapy currently. ${ }^{28}$ MRI has been historically regarded as the gold standard for CCMs diagnosis. CCMs can occur sporadically or be inherited in an autosomal dominant pattern. Mutations in one of three CCM genes, KRIT1/CCM1, $M G C 4607 / C C M 2$, and PDCD10/CCM3, have been predicted to cause loss of function. Here, we present a case report on a 44-year-old woman whose clinical and radiological phenotype was remarkable of CCMs. The Analyses toward the Whole Exome Sequencing revealed a novel disease-associated mutation in the already known diseaseassociated PDCD10 gene. This mutation consists of a nucleotide deletion (c.212delG) within the exon 4, resulting in premature protein termination (p.S71Tfs*18).

There are some significant features in the patient as following: (1) the patient's symptoms have been exacerbating during the progress of treatment. SWI sequence on 4/16/2018 showed more microhemorrhagic lesions and larger lesion size in the brain than SWI on 12/25/2017, which was the main reason for the progressively worsening headaches; 2) the patient with CCMs simultaneously suffered from multiple nodular skin lesions with a diameter of about $1 \mathrm{~cm}$, which was rare in CCMs; 3) about 150 different mutations have been found in $C C M 1$ leading to $\mathrm{CCMs}^{29}{ }^{29} 4$ different mutations in $C C M 2^{30}$ and 15 different mutations in $C C M 3$ have been identified in Western countries. ${ }^{31}$ It appears that $C C M 1$ is the dominant genetic cause of CCMs in Chinese people. To date, no loss- of-function mutations have been identified in $C C M 2$ or $C C M 3$ in china. Thus, this novel mutation in PDCD10 gene will be helpful to extensively understand the impact of $P D C D 10$ mutations on Chinese population and the difference between Chinese and Western.

Programmed cell death $10(P D C D 10)$ located on $3 \mathrm{q} 26.1$ is the third CCM locus and is also called CCM3. CCM3 identified more recently is a highly conserved gene including seven coding and three non-coding exons, which code a 212-amino acid protein $(P D C D 10){ }^{32}$ The protein, with an N-terminal dimerization domain and a $\mathrm{C}$-terminal focal adhesion targeting (FAT)-homology domain, ${ }^{33}$ is ubiquitously expressed. PDCD10 protein can interact with a variety of proteins including cell adhesion molecule Paxillin and Malcavernin (CCM2) through its C-terminal and also interact with Germinal Centre Kinase III (GCK III kinases) through its N-terminal, ${ }^{34}$ promoting cell proliferation, modulating apoptotic pathways and increasing mitogen-activated protein kinase (MAPK) activity and STK26 activity. ${ }^{35}$ In addition, a fully folded CCM3 FAThomology domain is vital for the stabilization of the expressed protein. ${ }^{36}$ Loss-of-function mutations in PDCD10 will lead to the onset of CCMs. ${ }^{37,38}$

So far, three known loci have accounted for $96 \%$ of CCM families. The frequency of identified mutations in the PDCD10 gene is surprisingly low to $10 \%(3 / 29)$, especially given that this panel is heavily biased towards non-CCM1, non-CCM2 probands. ${ }^{39}$ However, $P D C D 10$ has a high proportion of de novo mutations. Furthermore, the clinical features of CCMs in KRIT1, CCM2, and PDCD10 families have special differences. Patients with $C C M 3$ mutations are prone to have an earlier age at symptoms' onset and a higher risk of cerebral hemorrhage during childhood. ${ }^{23}$ Although there are three genes responsible for CCMs, recent studies have demonstrated that the $C C M 1$ and $C C M 2$ proteins interact with each other and are part of a common signaling complex. ${ }^{40}$ CCM2 contains a phosphotyrosine-binding domain that can bind to CCM1, which can affect the p38 MAPK signaling cascade. ${ }^{41}$ PDCD10 also binds its C-terminal directly to $C C M 2$ as well 
as several other signaling molecules. ${ }^{42} \mathrm{~A}$ recent research showed that CCM3 interacts with malcavernin, forming a part of the Krit1-malcavernin complex that participates in $C C M 1$-dependent $\beta 1$-integrin-mediated signal modulation and CCM2-mediated p38 MAPK signaling in response to cellular stress. $^{43}$ Thence, the interactions of CCM1, CCM2, and CCM3 cooperatively contribute to the hypothesis that they function as a complex to affect a common signaling mechanism. ${ }^{44,45}$ The hypothesis has already been proved by animal models. In the endothelium, both KRIT1 and CCM2 suppress the activity of the small GTPase RhoA. Deficient of CCM1 or CCM2 leads to RhoA activation and signal pathway through Rho kinase (ROCK), increasing actin stress fibers, impairing cell-cell interactions, increasing vascular permeability, and finally hindering the formation of the first branchial arch artery in mice. ${ }^{46}$ A recent study showed a similar mechanism of Rho activation for PDCD $10 .{ }^{47}$ However, other signal pathways of PDCD10 have also been studied. Advancements in genetic engineering and stem cell research will provide great potential for the interventional options for CCMs in the near future, ${ }^{48}$ which can lead to further understanding about CCMs.

This novel mutation significantly enriches the spectrum of mutations responsible for CCMs, providing a new evidence for further clarifying the genotype-phenotype correlations in CCMs patients. However, there are also many questions remain to be solved. First, it is pity that none of her family members could be investigated for the presence of CCMs; second, we still have not figured out the mechanism of how this novel mutation causes CCMs. Therefore, further laboratory studies and clinical assessment are necessary to identify potential targets for pharmacologic therapy.

\section{Author contributions}

All authors contributed toward data analysis, drafting and critically revising the paper, gave final approval of the version to be published, and agree to be accountable for all aspects of the work.

\section{Disclosure}

The authors report no conflicts of interest in this work.

\section{References}

1. Bicalho VC, Bergmann A, Domingues F, Frossard JT, de Souza J. Cerebral cavernous malformations: patient-reported outcome validates conservative management. Cerebrovasc Dis. 2017;44:313-319. doi:10.1159/000480125

2. Thompson K, Mattern-Baxter K. Intensive, functional training leads to optimal outcomes in a young woman post brain stem hemorrhage due to cerebral cavernous malformation. Physiother Theor Pract. 2018;1-12.
3. Tu J, Stoodley MA, Morgan MK, Storer KP. Ultrastructural characteristics of hemorrhagic, nonhemorrhagic, and recurrent cavernous malformations. J Neurosurg. 2005;103:903-909. doi:10.3171/jns.2005.103.5.0903

4. Zabramski JM, Wascher TM, Spetzler RF, et al. The natural history of familial cavernous malformations: results of an ongoing study. $J$ Neurosurg. 1994;80:422-432. doi:10.3171/ jns.1994.80.3.0422

5. Baranoski JF, Kalani MYS, Przybylowski CJ, Zabramski JM. Cerebral cavernous malformations: review of the genetic and protein-protein interactions resulting in disease pathogenesis. Front Surg. 2016;3. doi:10.3389/fsurg.2016.00060

6. Oka M, Sakaguchi M, Fukumoto T, Seki S, Nishigori C. Rare case of vascular malformations in both skin and brain: case report and published work review. $J$ Dermatol. 2017;44:813-817. doi:10.1111/13468138.13799

7. Yang C, Nicholas VH, Zhao J, et al. A novel CCM1/KRIT1 heterozygous nonsense mutation (c. $1864 \mathrm{C}>\mathrm{T}$ ) associated with familial cerebral cavernous malformation: a genetic insight from an 8-year continuous observational study. J Mol Neurosci. 2017;61:511-523. doi:10.1007/s12031-017-0893-1

8. Akers A, Al-Shahi SR, Awad AI, et al. Synopsis of guidelines for the clinical management of cerebral cavernous malformations: consensus recommendations based on systematic literature review by the angioma alliance scientific advisory board clinical experts panel. Neurosurgery. 2017;80:665-680. doi:10.1093/neuros/nyx091

9. Batra S, Lin D, Recinos PF, Zhang J, Rigamonti D. Cavernous malformations: natural history, diagnosis and treatment. Nat Rev Neurol. 2009;5:659-670. doi:10.1038/nrneurol.2009.177

10. Robinson JR, Awad IA, Little JR. Natural history of the cavernous angioma. J Neurosurg. 1991;75:709. doi:10.3171/jns.1991.75.5.0709

11. Rigamonti D, Hadley MN, Drayer BP, et al. Cerebral cavernous malformations. Incidence and familial occurrence. $N$ Engl $J$ Med. 1988;319:343-347. doi:10.1056/NEJM198808113190605

12. Coban A, Gurses C, Bilgic B, et al. Sporadic multiple cerebral cavernomatosis: report of a case and review of literature. Neurologist. 2008;14:46-49. doi:10.1097/NRL.0b013e31813e343f

13. Mosca L, Pileggi S, Avemaria F, et al. De novo MGC4607 gene heterozygous missense variants in a child with multiple cerebral cavernous malformations. $J$ Mol Neurosci. 2012;47:475-480. doi:10.1007/s12031-012-9741-5

14. Riant F, Bergametti F, Ayrignac X, Boulday G, Tournier-Lasserve E. Recent insights into cerebral cavernous malformations: the molecular genetics of CCM. FEBS J. 2010;277:1070-1075. doi:10.1111/j.17424658.2009.07535.x

15. Rosário Marques I, Antunes F, Ferreira N, Grunho M. Familial cerebral cavernous malformation: report of a novel KRIT1 mutation in a Portuguese family. Seizure. 2017;53:72-74. doi:10.1016/j. seizure.2017.10.020

16. Yang $\mathrm{C}$, Wu B, Zhong H, Li Y, Zheng X, Xu Y. A novel CCM1/ KRIT1 heterozygous deletion mutation (c.1919delT) in a Chinese family with familial cerebral cavernous malformation. Clin Neurol Neurosurg. 2018;164:44-46. doi:10.1016/j. clineuro.2017.11.005

17. Denier C, Goutagny S, Labauge P, et al. Mutations within the MGC4607 gene cause cerebral cavernous malformations. Am J Hum Genet. 2004;74:326-337. doi:10.1086/381718

18. Bergametti F, Denier C, Labauge P, et al. Mutations within the programmed cell death 10 gene cause cerebral cavernous malformations. Am J Hum Genet. 2005;76:42-51. doi:10.1086/426952

19. Cigoli MS, Avemaria F, De Benedetti S, et al. PDCD10 gene mutations in multiple cerebral cavernous malformations. PLoS One. 2014;9:e110438. doi:10.1371/journal.pone.0110438

20. Stahl S, Gaetzner S, Voss K, et al. Novel CCM1, CCM2, and CCM3 mutations in patients with cerebral cavernous malformations: in-frame deletion in CCM2 prevents formation of a CCM1/CCM2/CCM3 protein complex. Hum Mutat. 2008;29:709-717. doi:10.1002/humu.20712 
21. Zhu H, Guo Y, Feng X, et al. Familial cerebral cavernous angiomas: clinical and genetic features in a Chinese family with a frame-shift mutation in the CCM1 gene (krit1). $J$ Mol Neurosci. 2014;54:790-795. doi:10.1007/s12031-014-0415-3

22. Riant F, Bergametti F, Fournier HD, et al. CCM3 mutations are associated with early-onset cerebral hemorrhage and multiple meningiomas. Mol Syndromol. 2013. doi:10.1159/000350042

23. Denier C, Labauge P, Bergametti F, et al. Genotype-phenotype correlations in cerebral cavernous malformations patients. Ann Neurol. 2006;60:550-556. doi:10.1002/ana.20947

24. Shenkar R, Shi C, Rebeiz T, et al. Exceptional aggressiveness of cerebral cavernous malformation disease associated with PDCD10 mutations. Genet Med. 2015;17:188-196. doi:10.1038/gim.2014.97

25. Scimone C, Donato L, Esposito T, Rinaldi C, D'Angelo R, Sidoti A. A novel RLBP1 gene geographical area-related mutation present in a young patient with retinitis punctata albescens. Hum Genomics. 2017;11:18. doi:10.1186/s40246-017-0114-6

26. Clatterbuck RE, Eberhart CG, Crain BJ, Rigamonti D. Ultrastructural and immunocytochemical evidence that an incompetent blood-brain barrier is related to the pathophysiology of cavernous malformations. J Neurol Neurosurg Psychiatry. 2001;71:188-192.

27. Labauge P, Denier C, Bergametti F, Tournier-Lasserve E. Genetics of cavernous angiomas. Lancet Neurol. 2007;6:237-244. doi:10.1016/ S1474-4422(07)70053-4

28. Hasegawa T, McInerney J, Kondziolka D, Lee JY, Flickinger JC, Lunsford LD. Long-term results after stereotactic radiosurgery for patients with cavernous malformations. Neurosurgery. 2002;50:11901197, 1197-1198.

29. Cavé-Riant F, Denier C, Labauge P, et al. Spectrum and expression analysis of KRIT1 mutations in 121 consecutive and unrelated patients with cerebral cavernous malformations. Eur J Hum Genet. 2002;10:733-740. doi:10.1038/sj.ejhg.5200870

30. Scimone C, Donato L, Katsarou Z, Bostantjopoulou S, D'Angelo R, Sidoti A. Two novel KRIT1 and CCM2 mutations in patients affected by cerebral cavernous malformations: new information on CCM2 penetrance. Front Neurol. 2018;9:953. doi:10.3389/fneur.2018.00953

31. Scimone C, Bramanti P, Ruggeri A, et al. Detection of novel mutation in $\mathrm{Ccm} 3$ causes familial cerebral cavernous malformations. $J \mathrm{Mol}$ Neurosci. 2015;57:400-403. doi:10.1007/s12031-015-0606-6

32. Guclu B, Ozturk AK, Pricola KL, et al. Mutations in apoptosis-related gene, PDCD10, cause cerebral cavernous malformation 3. Neurosurgery. 2005;57:1008-1013.

33. Li X, Zhang R, Zhang H, et al. Crystal structure of CCM3, a cerebral cavernous malformation protein critical for vascular integrity. $J$ Biol Chem. 2010;285:24099-24107. doi:10.1074/jbc.M110.128470

34. Ceccarelli DF, Laister RC, Mulligan VK, et al. CCM3/PDCD10 heterodimerizes with germinal center kinase III (GCKIII) proteins using a mechanism analogous to CCM3 homodimerization. $J$ Biol Chem. 2011;286:25056-25064. doi:10.1074/jbc.M110.213777
35. Mardakheh FK, Self A, Marshall CJ. RHO binding to FAM65A regulates Golgi reorientation during cell migration. J Cell Sci. 2016;129:4466-4479. doi:10.1242/jcs.198614

36. Ding J, Wang X, Li D, Hu Y, Zhang Y, Wang D. Crystal structure of human programmed cell death 10 complexed with inositol-(1,3,4,5)tetrakisphosphate: A novel adaptor protein involved in human cerebral cavernous malformation. Biochem Bioph Res Co. 2010;399:587-592. doi:10.1016/j.bbrc.2010.07.119

37. Scimone C, Bramanti P, Alafaci C, et al. Update on novel CCM gene mutations in patients with cerebral cavernous malformations. $J \mathrm{Mol}$ Neurosci. 2017;61:189-198. doi:10.1007/s12031-016-0863-z

38. Scimone C, Bramanti P, Ruggeri A, et al. CCM3/SERPINI1 bidirectional promoter variants in patients with cerebral cavernous malformations: a molecular and functional study. BMC Med Genet. 2016;17:74. doi:10.1186/s12881-016-0332-0

39. Liquori CL, Berg MJ, Squitieri F, et al. Low frequency of PDCD10 mutations in a panel of CCM3 probands: potential for a fourth CCM locus. Hum Mutat. 2006;27:118. doi:10.1002/humu.9389

40. Zawistowski JS, Stalheim L, Uhlik MT, et al. CCM1 and CCM2 protein interactions in cell signaling: implications for cerebral cavernous malformations pathogenesis. Hum Mol Genet. 2005;14: 2521-2531. doi: $10.1093 / \mathrm{hmg} / \mathrm{ddi} 256$

41. Faurobert E, Albiges-Rizo C. Recent insights into cerebral cavernous malformations: a complex jigsaw puzzle under construction. FEBS J. 2010;277:1084-1096. doi:10.1111/j.1742-4658.2009.07537.x

42. Draheim KM, Li X, Zhang R, et al. CCM2-CCM3 interaction stabilizes their protein expression and permits endothelial network formation. $J$ Cell Biol. 2015;208:987-1001. doi:10.1083/ jcb.201407129

43. Zawistowski JS, Serebriiskii IG, Lee MF, Golemis EA, Marchuk DA. KRIT1 association with the integrin-binding protein ICAP-1: a new direction in the elucidation of cerebral cavernous malformations (CCM1) pathogenesis. Hum Mol Genet. 2002;11:389-396.

44. Hilder TL, Malone MH, Bencharit S, et al. Proteomic identification of the cerebral cavernous malformation signaling complex. J Proteome Res. 2007;6:4343-4355. doi:10.1021/pr0704276

45. Voss K, Stahl S, Schleider E, et al. CCM3 interacts with CCM2 indicating common pathogenesis for cerebral cavernous malformations. Neurogenetics. 2007;8:249-256. doi:10.1007/ s10048-007-0098-9

46. Whitehead KJ, Chan AC, Navankasattusas S, et al. The cerebral cavernous malformation signaling pathway promotes vascular integrity via Rho GTPases. Nat Med. 2009;15:177-184. doi:10.1038/nm.1911

47. Borikova AL, Dibble CF, Sciaky N, et al. Rho Kinase inhibition rescues the endothelial cell cerebral cavernous malformation phenotype. J Biol Chem. 2010;285:11760-11764. doi:10.1074/jbc. C109.097220

48. Li Y, Liu T, Van Halm-Lutterodt N, Chen J, Su Q, Hai Y. Reprogramming of blood cells into induced pluripotent stem cells as a new cell source for cartilage repair. Stem Cell Res Ther. 2016;7. doi:10.1186/s13287-016-0290-7
Journal of Pain Research

\section{Publish your work in this journal}

The Journal of Pain Research is an international, peer reviewed, open access, online journal that welcomes laboratory and clinical findings in the fields of pain research and the prevention and management of pain. Original research, reviews, symposium reports, hypothesis formation and commentaries are all considered for publication. The manuscript management system is completely online and includes a very quick and fair peer-review system, which is all easy to use. Visit http:// www.dovepress.com/testimonials.php to read real quotes from published authors. 